\title{
What are the key elements of youth sports orthopaedics examination?
}

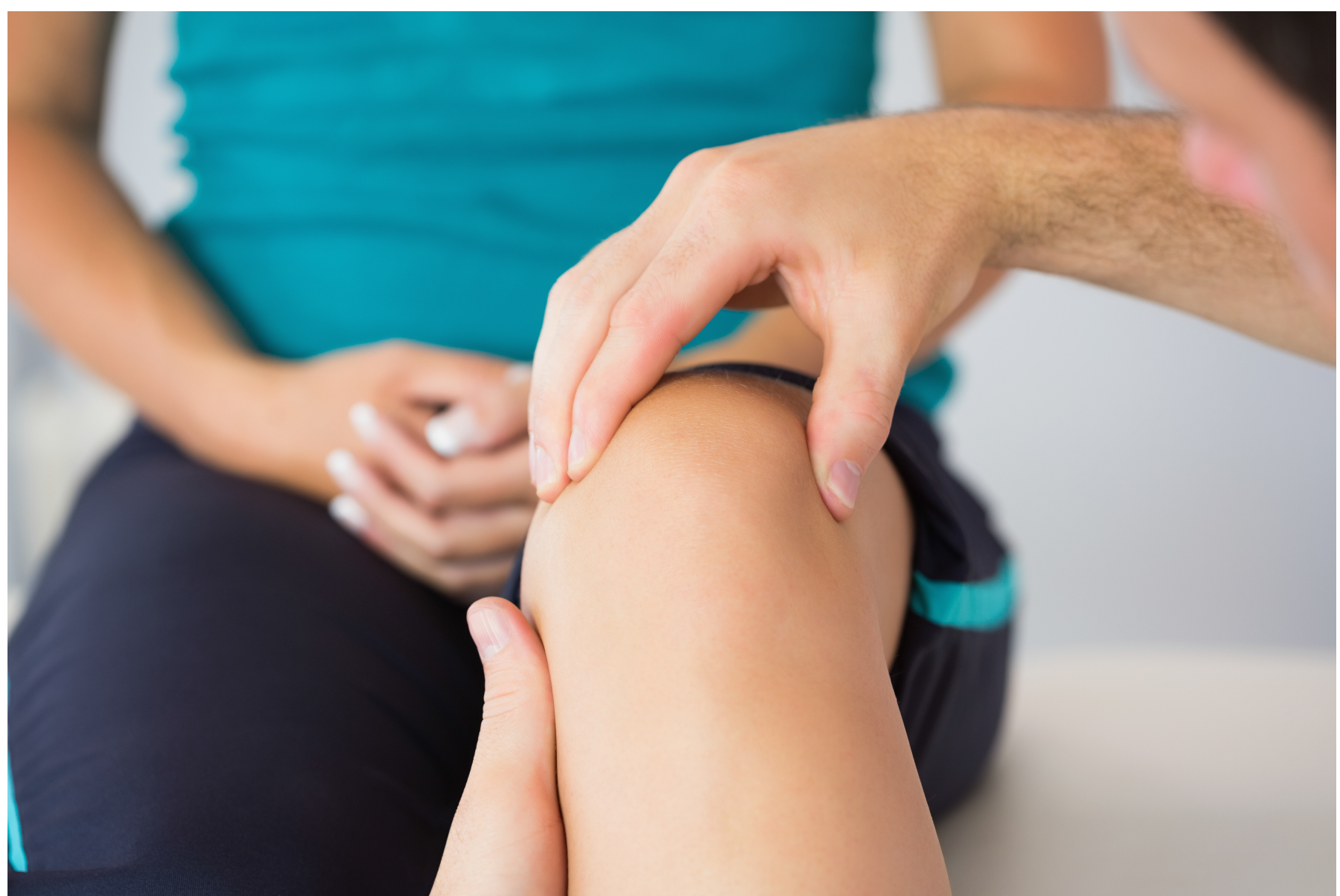

Wo ist der Fokus der sportorthopädischen Untersuchung bei Jugendlichen?

Camathias C, Studer K

Praxis Zeppelin, St. Gallen

\section{Abstract}

Young competitive athletes are particularly at risk during puberty. Growth plates and apophyses are reduced in their stability by hormone influence. Epiphyses can slip, apophyses can tear out. Therefore, a regular examination of those athletes is important. The examination should focus on muscular 
asymmetries, or reduced range of motion of a joint. Shortened muscles have to be recon as a risk factor for apophysitis. Dysbalances of the musculature are mainly found in the trunk area.

Training plans should be adapted to the increased vulnerability.

\section{Zusammenfassung}

Jugendliche Leistungssportler sind vor allem während der Pubertät gefährdet. Wachstumsfugen und Apophysen sind in ihrer Stabilität durch Hormoneinfluss vermindert. Epiphysen können verrutschen, Apophysen ausreissen. Der regelmässige Untersuch von jugendlichen Leistungssportlern ist deshalb wichtig. Der Untersuch sollte auf Asymmetrien der Muskulatur, aber auch Bewegungseinschränkungen der Gelenke fokussieren. Verkürzte Muskulaturen sind ein Risikofaktor für Probleme an Apophysen. Dysbalancen der Muskulatur sind vor allem im Rumpfbereich anzutreffen. Trainingspläne sollten der erhöhten Vulnerabilität angepasst werden.

\section{Einleitung}

Kinder und Jugendliche sind heutzutage häufiger sportlich aktiv. Nicht nur die Frequenz, sondern auch die Intensität dieser sportlichen Aktivitäten ist in den letzten Jahrzehnten angestiegen. Durch eine professionelle Betreuung der jungen Sportler, wird heutzutage auf hohem Niveau Leistungssport betrieben. Diese Entwicklung hin zum Leistungssport bedingt eine gute medizinische Betreuung, welche sich der speziellen Gegebenheiten im Kindes- und Jugendalter bewusst ist. Zu Beginn der Pubertät ändern sich meist die Trainingspläne der Sportler hin zu intensiven Belastungen. Nicht selten kann der Sport mehr als 20 Stunden pro Woche beanspruchen. In dieser Zeit steigt jedoch auch die Verletzlichkeit des Körpers. Am häufigsten sind Apophysen- und Fugenverletzungen zu verzeichnen. Das hängt von der erhöhten Belastung, einer erhöhten Risikobereitschaft der Jugendlichen und nicht zuletzt auch vom Einfluss der Hormone auf den Wachstumsknorpel ab. Vor allem Testosteron führt zu einer Erweichung der Fugen und Apophysen, welche in der Folge Scherkräften nicht mehr derart standhalten. Die Epiphyse droht abzurutschen, die Apophyse auszureissen. In unserer Vorstellung erscheinen die notwendigen Kräfte relativ hoch, um Schaden anzurichten. Kadaver-Modelle legen einen anderen Schluss nahe. Es reichen Kräfte, welche physiologischen Belastungen bei übergewichtigen Kindern entsprechen, um eine Epiphysiolyse zu provozieren. 


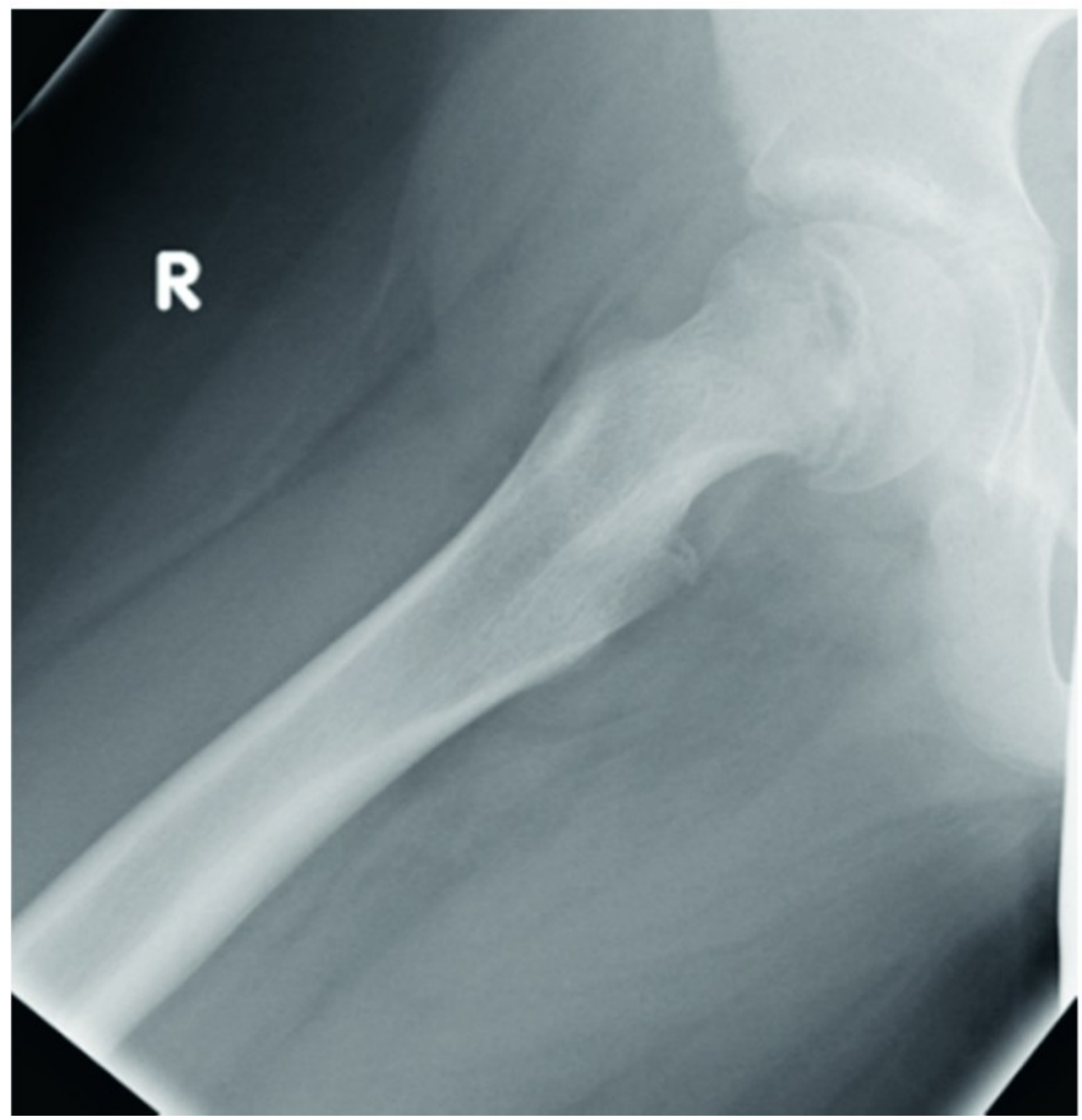

Abbildung 1: Epiphysiolyse capitis femoris.Häufig kann die Diagnose erst in einer zweiten Ebene im Röntgenbild dargestellt werden.

Die Epiphyseolysis capitis femoris ist der bekannteste Vertreter einer abgerutschten Epiphyse bzw. des Schenkelhalses (Abbildung 1). Wenn die Epiphysiolysis akut eintritt, ist die Diagnose meist problemlos zu stellen, da der Patient starke Schmerzen verspürt und nicht mehr gehfähig ist. Rutscht die Epiphyse hingegen langsam und chronisch, fällt der Schmerz nicht in jedem Fall auf. Kontinuierlich verschlechtert sich der Zustand der Hüfte - ein langwieriges Problem droht zu entstehen. Bei der Epiphyseolyis capitis - 
femoris ist das CAM-Impingement die bekannteste Folgeproblematik. Sie kann erst Jahrzehnte später zu einer Degeneration der Hüfte und zu behandlungsbedürftigen Schmerzen führen. Häufig ist der Ursprung solch eines CAM-Impingement auf dem Boden einer Epiphysiolyse entstanden und in der Jugendzeit ignoriert worden. Bei jungen Leistungssportlern wird ein leichter Hüftschmerz häufig toleriert und als normale Erscheinung des Trainings abgetan. So haben beispielsweise Eishockeyaner ein erhöhtes Risiko, später an einem CAM-Impingement zu erkranken. In vielen Fällen findet sich die Ursache in der Pubertät. Es macht deshalb Sinn, regelmässige orthopädische Untersuchungen bei sportlichen Jugendlichen durchzuführen, um zu beurteilen, ob der Körper korrekt belastet wird und es weiter kann. Damit sollen mögliche Risikofaktoren für spätere Verletzungen erkannt und minimiert werden. Selbstverständlich soll aus einer erkannten Gefahr auch eine Therapieempfehlung oder Prävention abgeleitet werden können. Grundsätzlich unterscheidet sich eine sportorthopädische Untersuchung bei Jugendlichen und Kinder, nicht sonderlich von jener bei Erwachsenen. Trotzdem sollte auf einen kindgerechten Umgang geachtet werden.

Untersucht man Jugendliche, müssen sie unter dem Aspekt möglicher Fugen- bzw. ApophysenÜberlastungen beurteilt werden. Aufhorchen sollte der Untersucher, wenn jugendliche Sportler über chronische Schmerzen im Bereiche von Aphophysen oder Gelenken klagen. Schwellungen in diesen Bereichen sind Zeichen für Überlastungen. Aufgrund des Wachstums tendieren Jugendliche in der Pubertät häufig zu verkürzter Muskulatur mit starkem Zug und Stress auf die Apophyse. Die Apophyse entzündet und schmerzt in der Folge. Typisches Beispiel für eine gestresste Apophyse ist der Morbus Osgood-Schlatter am Kniegelenk, oder die Apophysitis calcanei. An beiden Aphophysen sind meist verkürzte Muskulaturen zu finden. Zusätzlich sind auch Faszien bzw. das gesamte System verkürzt, sodass reines Dehnen der Muskulatur meist nicht allein zielführend ist.

Apophysitiden sind Ausdruck einer langsamen jedoch kontinuierlichen Überlastung. Diese Kontinuität entsteht vielfach bei einseitigem Training. Polysportive Jugendliche tendieren weniger zu Überlastungen mit Folgeproblemen. Überlastungen könnnen zunehmen, wenn zu einer einseitigen sportlichen Tätigkeit noch Schwächen der Muskulatur hinzukommen. Es ist nicht selten, dass Leistungssportler zwar über enorme Muskelpakete an den Beinen verfügen, den Rumpf jedoch nicht genügend stabilisieren können. Solche Muskel-Ungleichgewichte verursachen an den Muskelansätzen hohe Kraftspitzen, die an Apophysen chronische Beschwerden auslösen können. Tritt die Überlastung plötzlich auf, kann die gesamte Apophyse samt Muskel ausreissen. Ein typisches Beispiel hierfür ist der Ausriss des Musculus rectus femoris des Quadrizeps an der Spina iliaca anterior inferior am Becken. Meist besitzt der Verletzte einen ausgesprochen gut ausgebildeten Quadrizepsmuskel, welcher deutlich verkürzt ist - eine häufige Kombination bei Fussballern (Abbildung 2a). Werden diese Verletzungen übersehen, so kann es zu einer relevanten Kallusbildung kommen, welche wiederum ein sogenanntes Subspine-Impingement auslösen kann.

Die orthopädische Untersuchung fokussiert in diesem Alter beim beschwerdefreien Sportler vor allem auf die Muskulatur, bzw. deren Länge. Verkürzte Muskulatur bei hoher Trainingsbelastung sollte Anlass zu Prävention und Änderung des Trainingsplanes geben.

Die Untersuchung sollte entsprechend des durchgeführten Sports angepasst werden. Schulter-belastende Sportarten wie Handball, Tennis, Basketball usw. beurteilt man mit einem Fokus auf die obere Extremität. Fussballer, Laufsportler und Ähnliche zeigen vermehrt Probleme im Bereich der unteren Extremitäten. Nichtsdestotrotz sollte der gesamte Bewegungsapparat beurteilt werden. Den ersten Überblick verschafft 
man sich, indem man den Bewegungsumfang der grossen Gelenke misst und Seitendifferenzen feststellt. Jede Differenz bzw. Bewegungseinschränkung eines Gelenkes sollte Anlass zur profunden Untersuchung sein. Sodann sollten vor allem die grossen Muskulaturen, welche häufig zu Verkürzungen neigen, separat untersucht werden. So gehört die Beurteilung der Länge der Hamstrings, des Rectus femoris, der Gastrocnemiusmuskulatur zum Standard der unteren Extremität. Hier sind spezifische Tests bekannt wie beispielsweise der Duncan-Ely-Test für die Beurteilung der verkürzten Rectus femoris Muskulatur. Die Verkürzung wird dabei in Bauchlage getestet. Die Hüfte ist gestreckt, das Kniegelenk wird gebeugt. Hebt sich dabei das Becken von der Unterlage, ist der Rektus femoris verkürzt. Für die Hamstrings- und die Gastrocnemiusmuskulatur stehen ähnliche Tests zur Verfügung. Die Untersuchung sollte Asymmetrien der Muskulatur aufdecken. Hier sind Unterschiede von einer Extremität zur anderen zu suchen. Wichtiger hingegen ist, keine grosse Asymmetrie zwischen antagonistischer und agonistischer Muskulatur zu verpassen. Vor allem im Bereich des Rumpfes treten häufig Dysbalancen auf, welche bereits bei einem simplen Trendelenburg-Test auffallen können.

Treten im Untersuch Unregelmässigkeiten auf, beklagt der Patient Beschwerden, sollten diese vor allem in der Pubertät ernst genommen werden. Entsprechend sollte die Belastung reduziert oder das Belastungsmuster geändert werden. Um nicht den Frust des jungen Sportlers zu vergrössern, kann anstatt einer Verminderung auf andere Aktivitäten ausgewichen werden. Wichtig ist in diesem Zusammenhang der Einbezug der Eltern und der Trainer. Alle Parteien müssen darauf hingewiesen werden, dass sich der junge Sportler in einer vulnerablen Zeit befindet und nicht die sportliche Leistung in jedem Fall im Vordergrund stehen sollte. 


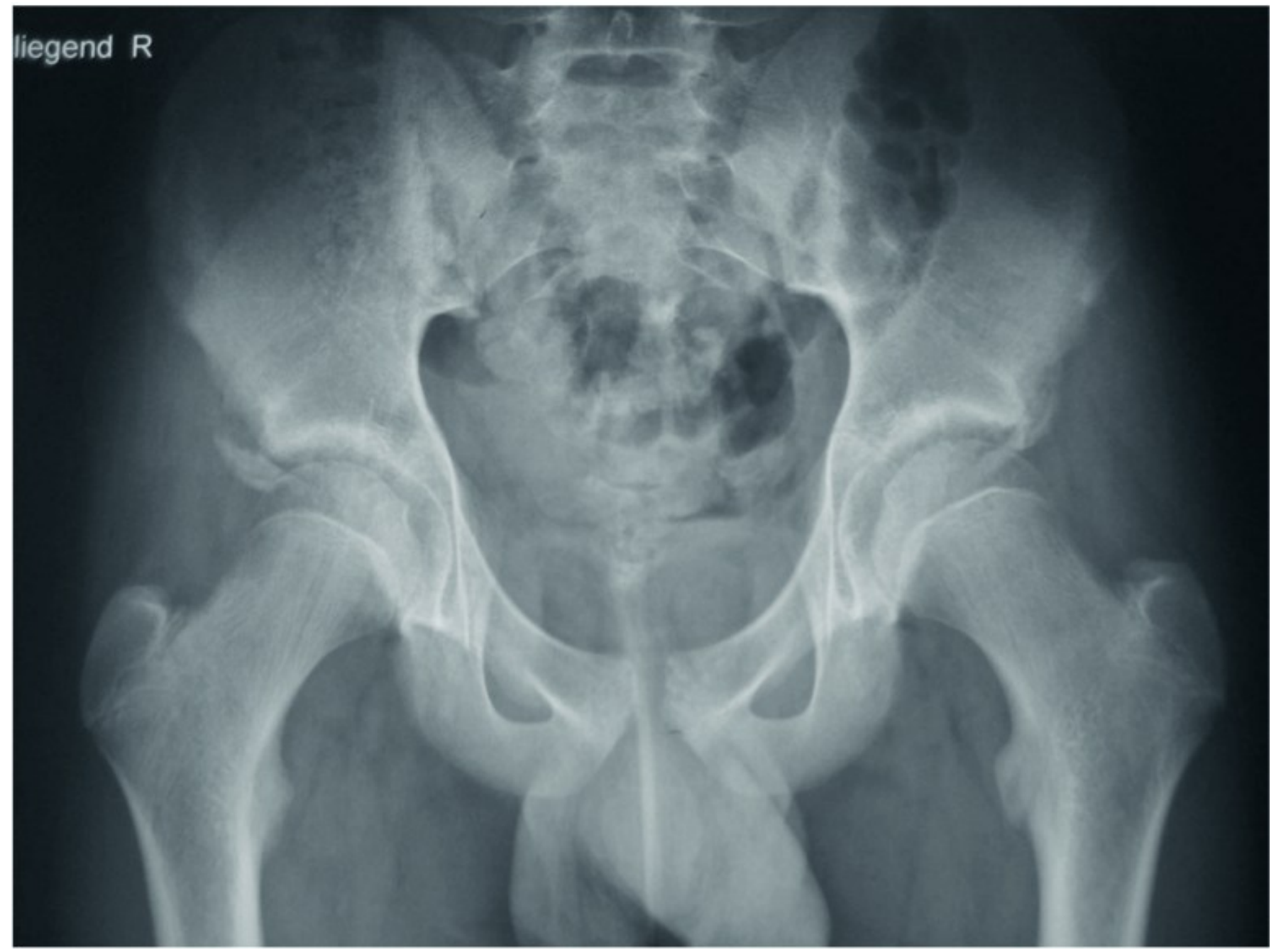

Abbildung 2a: Ausriss des M. Rectus femoris an der Spina iliaca anterior inferior. 


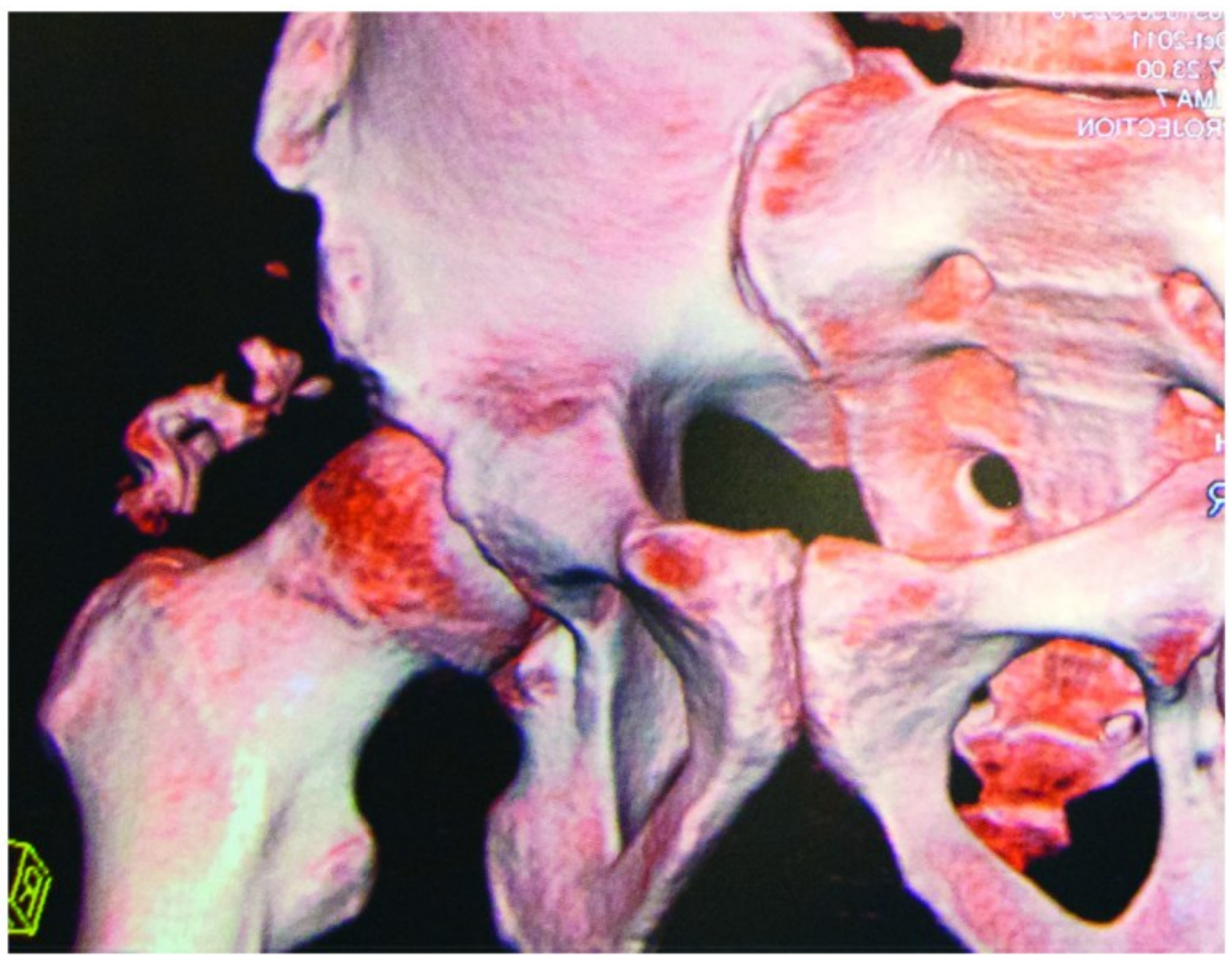

Abbildung 2b: Die Apophyse kann vergrössert verknöchern und zu einem Impigement an der Hüfte führen.

\section{Praktische Implikation}

Jugendliche in der Pubertät sind bezüglich Fugen- und Apophysenverletzungen gefährdet.

Der Untersuch sollte auf Asymmetrien der Muskulatur, aber auch Bewegungseinschränkungen der Gelenke fokussieren.

Trainingspläne sollten entsprechend der erhöhten Vulnerabilität angepasst werden.

\section{Interessenskonflikte}

Die Autoren haben keine Interessenskonflikte.

\section{Corresponding author}


PD Dr. med. Carlo Camathias

Tel: 0712828242

info.zeppelin@hin.ch

http://www.praxis-zeppelin.ch

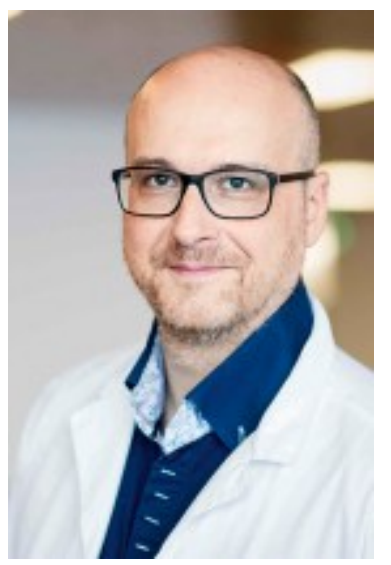

\section{Referenzen}

Alle Referenzen sind bei den Autoren.

APOPHYSES COMPETITIVE ATHLETES EPIPHYSEAL JOINTS YOUTH 